\title{
Assessment of backgrounds of the ANAIS experiment for dark matter direct detection
}

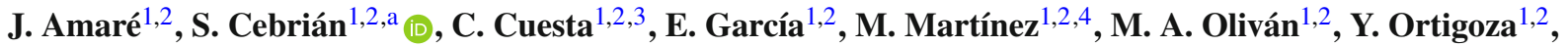 \\ A. Ortiz de Solórzano ${ }^{1,2}$, J. Puimedón ${ }^{1,2}$, M. L. Sarsa ${ }^{1,2}$, J. A. Villar ${ }^{1,2}$, P. Villar ${ }^{1,2}$ \\ ${ }^{1}$ Laboratorio de Física Nuclear y Astropartículas, Universidad de Zaragoza, Calle Pedro Cerbuna 12, 50009 Zaragoza, Spain \\ ${ }^{2}$ Laboratorio Subterráneo de Canfranc, Paseo de los Ayerbe s/n, Canfranc Estación, 22880 Huesca, Spain \\ ${ }^{3}$ Present Address: Department of Physics, Center for Experimental Nuclear Physics and Astrophysics, University of Washington, Seattle, WA, \\ USA \\ ${ }^{4}$ Present Address: Università di Roma La Sapienza, Piazzale Aldo Moro 5, 00185 Rome, Italy
}

\begin{abstract}
A large effort has been carried out to characterize the background of sodium iodide crystals within the Annual modulation with NaI Scintillators (ANAIS) project. In this paper, the background models developed for three $12.5 \mathrm{~kg} \mathrm{NaI}(\mathrm{Tl})$ scintillators produced by Alpha Spectra Inc. and operated at the Canfranc Underground Laboratory are presented together with an evaluation of the background prospects for the full experiment. Measured spectra from threshold to high energy in different conditions are well described by the models based on quantified activities. At the region of interest, crystal bulk contamination is the dominant background source. Contributions from ${ }^{210} \mathrm{~Pb},{ }^{40} \mathrm{~K},{ }^{22} \mathrm{Na}$ and ${ }^{3} \mathrm{H}$ are the most relevant. Those from ${ }^{40} \mathrm{~K}$ and ${ }^{22} \mathrm{Na}$ could be efficiently suppressed thanks to anticoincidence operation in a crystals matrix or inside a liquid scintillator veto (LSV), while that from ${ }^{210} \mathrm{~Pb}$ has been reduced by improving crystal production methods and ${ }^{3} \mathrm{H}$ production could be reduced by shielding against cosmic rays during production. Assuming the activities of the last characterized detector, for nine crystals with a total mass of $112.5 \mathrm{~kg}$ the expected background rate is 2.5 counts/( $\mathrm{keV} \mathrm{kg}$ day) in the region from 1 to $4 \mathrm{keV}$, which could be reduced at 1.4 counts/(keV kg day) by using a LSV.
\end{abstract}

\section{Introduction}

Sodium iodide crystals doped with $\mathrm{Tl}$ have been widely used as radiation detectors and, in particular, they have been applied in the direct search of galactic dark matter particles for a long time [1-5]. Among the several experimental

a e-mail: scebrian@unizar.es approaches using $\mathrm{NaI}(\mathrm{Tl})$ detectors, DAMA/ LIBRA is the most relevant, having reported the observation of a modulation compatible with that expected for galactic halo WIMPs with a large statistical significance [6]. Results obtained with other target materials and detection techniques (like those from CDMS [7], CRESST [8], EDELWEISS [9], KIMS [10], LUX [11], PICO [12] or XENON [13] collaborations) have been ruling out for years the most plausible compatibility scenarios. The Annual modulation with $\mathrm{NaI}$ Scintillators (ANAIS) project [14] is intended to search for dark matter annual modulation with ultrapure $\mathrm{NaI}(\mathrm{Tl})$ scintillators at the Canfranc Underground Laboratory (LSC) in Spain. The aim is to provide a model-independent confirmation of the annual modulation positive signal reported by DAMA/LIBRA using the same target and technique, but different experimental conditions affecting systematics. Projects like DM-Ice [15], KIMS [16] and SABRE [17] also envisage the use of large masses of $\mathrm{NaI}(\mathrm{Tl})$ for dark matter searches.

ANAIS aims at the study of the annual modulation signal using a $\mathrm{NaI}(\mathrm{Tl})$ mass of $112.5 \mathrm{~kg}$ at the LSC. To confirm the DAMA/LIBRA results, ANAIS detectors should be comparable to those of DAMA/LIBRA in terms of energy threshold and radioactive background: energy threshold lower than $2 \mathrm{keVee}^{1}$ and background at $1-2$ counts/(keV kg day) in the region of interest (RoI) below 6 keVee. Several prototypes have been developed and operated at LSC using BICRON and Saint-Gobain crystals; all of them were disregarded due to an unacceptable K content in the crystal at the level of hundreds of ppb. Among them, the so-called ANAIS-0 detector [18-21], a $9.6 \mathrm{~kg}$ Saint-Gobain crystal similar to those of DAMA experiment, has to be highlighted because its suc-

\footnotetext{
${ }^{1}$ Electron equivalent energy.
} 
cessful background model [18] has been the starting point for the present work. Some other interesting results, as very slow scintillation in $\mathrm{NaI}(\mathrm{Tl})$ [20] or an anomalous fast event population attributable to quartz light emission [22] were also obtained from first prototypes. The main challenge for ANAIS has been the achievement of the required low background level, being contaminations in the bulk of the crystal the dominant contribution in the RoI.

The new prototypes built by Alpha Spectra Inc. consist of $12.5 \mathrm{~kg} \mathrm{NaI}(\mathrm{Tl})$ crystals, housed in Oxygen Free Electronic (OFE) copper and coupled through quartz windows to two Hamamatsu photomultipliers (PMTs) at the LSC clean room in a second step; they have been fully tested and characterized at the LSC since the end of 2012, obtaining very promising results.

In the full ANAIS experiment, the total $\mathrm{NaI}(\mathrm{Tl})$ active mass will be divided into a number of such modules: nine of them, accounting for $112.5 \mathrm{~kg}$, will be set-up at LSC along 2016. The shielding for the experiment will consist of $10 \mathrm{~cm}$ of archaeological lead, $20 \mathrm{~cm}$ of low activity lead, $40 \mathrm{~cm}$ of neutron moderator, an anti-radon box (to be continuously flushed with radon-free air) and an active muon veto system made up of plastic scintillators designed to cover top and sides of the whole ANAIS set-up. The hut that will house the experiment at the hall B of LSC (under 2450 m.w.e.) is already operative, shielding materials and electronic chain components are prepared for mounting [23]. Different PMT models were tested in order to choose the best option in terms of light collection and background [24]. The Hamamatsu R12669SEL2 was selected and all the required units are available at the LSC.

The construction of reliable background models is essential for experiments demanding ultra low background conditions since they provide guidance and constraints for design and for analyzing any possible systematics and allow robust estimations of the experiment sensitivity (see some recent examples at [25-30]). It is worth noting that the reliability of this kind of studies depends on three important aspects: an accurate assay of background sources, a careful computation of their contribution to the experiment (typically made by Monte Carlo simulation) and continuous validation of the obtained results against experimental data, which will be stronger if data in different experimental conditions and energy ranges are considered. On the other hand, a complete understanding of the DAMA/LIBRA background at low energy has not yet been achieved and some open questions remain $[31,32]$. Therefore, a careful analysis and quantification of the different background components in the ANAIS prototypes produced by Alpha Spectra was undertaken and is presented here. The quantification of cosmogenic radionuclide production and its effects in $\mathrm{NaI}(\mathrm{Tl})$ crystals using data from the two first prototypes (D0 and D1, see Sect. 5.1) have been specifically studied at [33].
The structure of the article is the following. The experimental set-ups of the detectors and the measurements taken are described in Sect. 2. Sections 3 and 4 present the background sources considered and the details of their simulation. The quantified background contributions and the comparison with data at different energy ranges and experimental conditions are discussed in Sect. 5. Finally, the background projections for the full ANAIS experiment based on the obtained results with the first modules are shown in Sect. 6, while conclusions are summarized in Sect. 7.

\section{Experimental set-ups}

Two prototypes of $12.5 \mathrm{~kg}$ mass (named D0 and D1), made by Alpha Spectra (AS) Inc., CO (US), with ultrapure NaI powder, took data at the LSC from December 2012 to March 2015; we will refer in the following to this set-up as ANAIS25 [34]. Its main goals were to measure the crystal internal contamination, determine light collection, fine tune the data acquisition and test the filtering and analysis protocols.

The ANAIS-37 set-up $[35,36]$ combines the ANAIS-25 modules with a new one (named D2) also built by AS, using improved protocols in order to prevent radon contamination and WIMPScint-II grade powder. The crystal was received on the 6th of March, 2015 and data taking started five days later. Data considered in this work were taken from March to September 2015. The new module (D2) was placed in between the two ANAIS-25 modules (D0 and D1) to maximize the coincidence efficiency for the potassium determination (see Fig. 1). The main goal of ANAIS-37 set-up was to characterize the new D2 module, in particular, to evaluate the reduction of ${ }^{210} \mathrm{~Pb}$ contamination, to check the content of ${ }^{40} \mathrm{~K}$ and ${ }^{238} \mathrm{U}$ and ${ }^{232} \mathrm{Th}$ chains and to assess also its general performance.

The three AS modules are cylindrical, 4.75" diameter and $11.75^{\prime \prime}$ length, with synthetic quartz windows for PMTs coupling; they were encapsulated following similar protocols and using the same materials. Hamamatsu R12669SEL2 PMTs were coupled at LSC clean room for the three detectors. A Mylar window in the lateral face allows for low energy calibration. The shielding in both set-ups consisted of $10 \mathrm{~cm}$ of archaeological lead plus $20 \mathrm{~cm}$ of low activity lead shielding inside a radon exclusion box at LSC. An active muon veto system made up of plastic scintillators covered the top and sides of the shielding, first the coverage was only partial, but at the end of the ANAIS-37 data taking period the veto was fully operative.

Concerning the data acquisition system of these set-ups, each PMT charge output signal is separately processed for obtaining trigger, pulse shape digitization and energy at different ranges. Triggering is done by the coincidence (logical AND) of the two PMT signals of any detector at photoelec- 


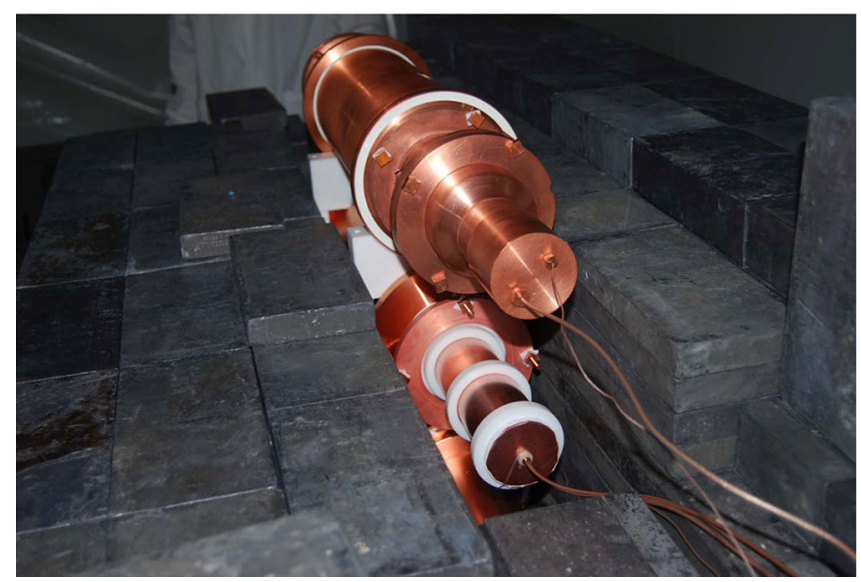

Fig. 1 Picture (left) and design (right) of the ANAIS-37 set-up at LSC

tron level in a $200 \mathrm{~ns}$ window, enabling digitization and conversion of the two signals. The building of the spectra is done by software (off-line) by adding the signals from both PMTs, and Pulse Shape Analysis is applied in order to select bulk scintillation events in the NaI crystals and to distinguish alpha interactions from beta/gamma ones. Data considered here correspond to the low and high energy regions, below $200 \mathrm{keV}$ and up to $3 \mathrm{MeV}$, respectively. Filtering protocols for PMT noise similar to those described at [21] for ANAIS0 prototype but optimized for these new detectors have been applied, and a threshold at $1 \mathrm{keVee}$ has been considered in the following, although work is still ongoing in order to improve the filtering and the acceptance efficiency estimate, probably underestimated at present [14].

Regarding the response of the detectors, it must be remarked the outstanding light collection measured for the three AS modules, at the level of $\sim 15 \mathrm{phe} / \mathrm{keV}$ [37], which is a factor of 2 larger than that determined for the best DAMA/LIBRA detectors [38]. This much higher light output has a direct impact in both resolution and energy threshold, but it also allows to improve strongly the signal vs noise filtering down to the threshold and hence, reduce analysis uncertainties.

\section{Background contributions}

The background sources considered for the ANAIS prototypes include activities from crystals as well as from external components. The latter have been mainly directly assayed by HPGe spectrometry at LSC. Table 1 summarizes the measured activities (or derived upper limits) of the components used in the set-up and taken into consideration. Every PMT unit to be used in the full experiment has been already

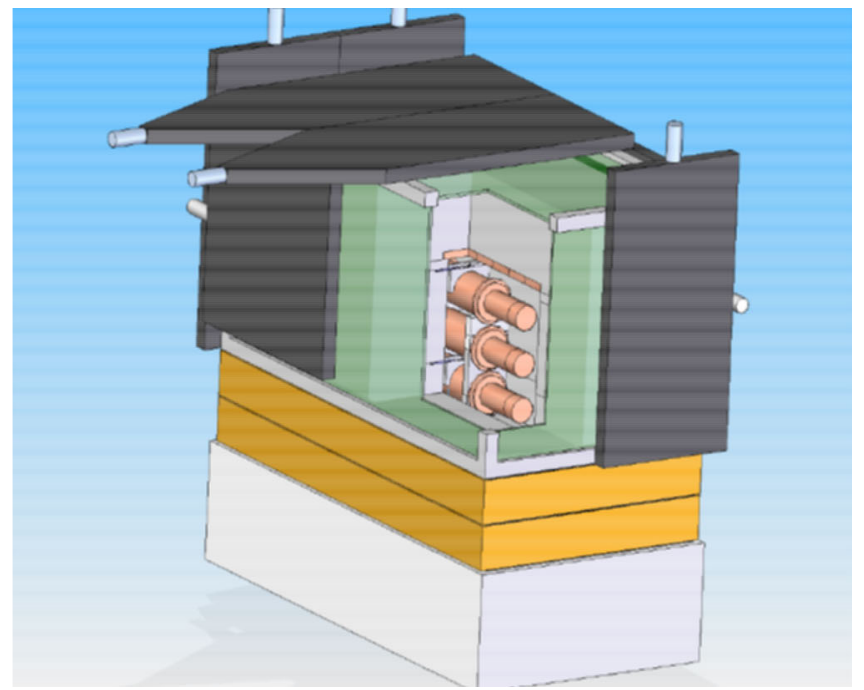

screened, finding compatible levels of activity among them; values quoted in Table 1 correspond to the six units used in the ANAIS-25 and ANAIS-37 set-ups and to the mean from all screened units. For copper and quartz windows values are as for ANAIS-0 prototype [18]. For radon content in the air filling the inner volume of the shielding, there is no direct measurement. Radon in the laboratory air is being continuously monitored, and the inner volume of the shielding is flushed with boil-off nitrogen, to guarantee its radon-free quality. A value for the radon content in the inner volume air of about one hundredth of the external air radon content has been assumed in our background model $\left(0.6 \mathrm{~Bq} / \mathrm{m}^{3}\right)$, compatible with the absence of lines coming from radon daughter isotopes in the measured background. Contribution from fast neutrons and environmental gamma background has been also estimated, being negligible at the present level of sensitivity. Contribution from muons interacting in the crystal (and other muon related events) can be vetoed by the coincidence with a signal in the plastic scintillators covering the shielding and then, it has not been considered in our background model. Although the active veto was not in operation during the data taking this work refers to, the muon induced background is negligible [21].

Concerning the background from the $\mathrm{NaI}(\mathrm{Tl})$ crystals, the activity of the most relevant radionuclides has been directly measured for our crystals applying different techniques [34]. Bulk ${ }^{40} \mathrm{~K}$ content is estimated by searching for the coincidences between $3.2 \mathrm{keV}$ energy deposition in one detector (following EC) and the $1461 \mathrm{keV}$ gamma line escaping from it and being fully absorbed in other detector [19]; efficiency of the coincidence is estimated by Monte Carlo simulation. The activities from ${ }^{210} \mathrm{~Pb}$ and ${ }^{232} \mathrm{Th}$ and ${ }^{238} \mathrm{U}$ chains have been determined on the one hand, by quantifying $\mathrm{Bi} / \mathrm{Po}$ and alpha-alpha sequences, and on the other, by comparing the 
Table 1 Activity of the external components (outside crystal) of the ANAIS prototypes considered as background sources. Except for the inner volume air, the values have been measured by HPGe spectrometry performed at the LSC. Upper limits are given at $95 \%$ C.L

\begin{tabular}{|c|c|c|c|c|c|c|}
\hline Component & Unit & ${ }^{40} \mathrm{~K}$ & ${ }^{232} \mathrm{Th}$ & ${ }^{238} \mathrm{U}$ & ${ }^{226} \mathrm{Ra}$ & Others \\
\hline \multirow[t]{6}{*}{ PMTs (R12669SEL2) } & \multirow[t]{6}{*}{$\mathrm{mBq} / \mathrm{PMT}$} & $97 \pm 19$ & $20 \pm 2$ & $128 \pm 38$ & $84 \pm 3$ & \\
\hline & & $133 \pm 13$ & $20 \pm 2$ & $150 \pm 34$ & $88 \pm 3$ & \\
\hline & & $108 \pm 29$ & $21 \pm 3$ & $161 \pm 58$ & $79 \pm 5$ & \\
\hline & & $95 \pm 24$ & $22 \pm 2$ & $145 \pm 29$ & $88 \pm 4$ & \\
\hline & & $136 \pm 26$ & $18 \pm 2$ & $187 \pm 58$ & $59 \pm 3$ & \\
\hline & & $155 \pm 36$ & $20 \pm 3$ & $144 \pm 33$ & $89 \pm 5$ & \\
\hline Mean activity all units & $\mathrm{mBq} / \mathrm{PMT}$ & $111 \pm 5$ & $20.7 \pm 0.5$ & $157 \pm 8$ & $82.5 \pm 0.8$ & \\
\hline Copper encapsulation & $\mathrm{mBq} / \mathrm{kg}$ & $<4.9$ & $<1.8$ & $<62$ & $<0.9$ & ${ }^{60} \mathrm{Co}:<0.4$ \\
\hline Quartz windows & $\mathrm{mBq} / \mathrm{kg}$ & $<12$ & $<2.2$ & $<100$ & $<1.9$ & \\
\hline Silicone pads & $\mathrm{mBq} / \mathrm{kg}$ & $<181$ & $<34$ & & $51 \pm 7$ & \\
\hline Archaeological lead & $\mathrm{mBq} / \mathrm{kg}$ & & $<0.3$ & $<0.2$ & & ${ }^{210} \mathrm{~Pb}:<20$ \\
\hline Inner volume air & $\mathrm{Bq} / \mathrm{m}^{3}$ & & & & & ${ }^{222} \mathrm{Rn}: 0.6$ \\
\hline
\end{tabular}

Table 2 Measured activity in $\mathrm{NaI}(\mathrm{Tl})$ crystals for D0, D1 and D2 detectors using ANAIS-25 and ANAIS-37 data and combining different analysis techniques

\begin{tabular}{llllll}
\hline Detector & Unit & ${ }^{40} \mathrm{~K}$ & ${ }^{232} \mathrm{Th}$ & ${ }^{238} \mathrm{U}$ & ${ }^{210} \mathrm{~Pb}$ \\
\hline D0, D1 & $\mathrm{mBq} / \mathrm{kg}$ & $1.4 \pm 0.2(\mathrm{D} 0)$ & $(4 \pm 1) 10^{-3}$ & $(10 \pm 2) 10^{-3}$ & $3.15 \pm 0.10$ \\
& & $1.1 \pm 0.2(\mathrm{D} 1)$ & & & \\
D2 & $\mathrm{mBq} / \mathrm{kg}$ & $1.1 \pm 0.2$ & $(0.7 \pm 0.1) 10^{-3}$ & $(2.7 \pm 0.2) 10^{-3}$ & $0.70 \pm 0.10$ \\
\hline
\end{tabular}

total alpha rate determined through pulse shape analysis with the low energy depositions attributable to ${ }^{210} \mathrm{~Pb}$, which are fully compatible. The results obtained for D0 and D1 detectors using ANAIS-25 data (see Table 2) gave a moderate contamination of ${ }^{40} \mathrm{~K}$, above the initial goal of ANAIS (20 ppb of K) but acceptable, a low content of ${ }^{232} \mathrm{Th}$ and ${ }^{238} \mathrm{U}$ chains but a high activity of ${ }^{210} \mathrm{~Pb}$ at the $\mathrm{mBq} / \mathrm{kg}$ level. The origin of such contamination was identified and addressed by Alpha Spectra. According to preliminary results from ANAIS-37 corresponding to 168.1 days of live time and shown in Table 2 , an average total alpha activity of $0.70 \pm 0.10 \mathrm{mBq} / \mathrm{kg}$ has been observed for the new module D2, which is a factor 5 lower than the alpha activity in ANAIS-25 modules; therefore, it can be concluded that effective reduction of $\mathrm{Rn}$ entrance in the growing and/or purification at Alpha Spectra has been achieved and is expected to improve for next prototypes. The potassium content of D2 was analyzed using the same technique applied to previous prototypes, obtaining a value compatible with those obtained for D0 and D1. The potassium concentration and alpha activity reported by the KIMS collaboration for a similar crystal produced also by Alpha Spectra from WIMPScint-II material [39] are of the same order than those measured for D2.

Thanks to the very good detector response and the prompt data taking starting after storing the detectors underground, a detailed study of cosmogenic radionuclide production in $\mathrm{NaI}(\mathrm{Tl})$ has been performed from ANAIS-25 data [33]. The initial activity, $A_{0}$, corresponding to the moment of storing crystals deep underground at LSC was deduced, studying the exponential decay of the identifying signature produced by each isotope. The crystal growing and detector manufacture took place at Alpha Spectra facilities in Grand Junction, CO (US) and detectors were taken from US to Spain by boat. The production of some induced I, Te and $\mathrm{Na}$ isotopes was well characterized and it was considered as a background source of the detectors too. Table 3 shows the list of all identified products and their half-lives, together with the measured initial activities. ${ }^{22} \mathrm{Na}$ could be specially worrisome for dark matter searches because the binding energy of the K-shell of its daughter $\mathrm{Ne}$ is $0.87 \mathrm{keV}$, falling the corresponding energy deposition in the RoI, and having a long enough half-life to compromise the first years of data taking. A direct estimate of ${ }^{22} \mathrm{Na}$ activity in D2 crystal was carried out by analyzing coincidences ${ }^{2}$; the obtained value for the initial activity, $A_{0}=(70.2 \pm 3.9) \mathrm{kg}^{-1} \mathrm{day}^{-1}$, is more than a factor of two lower than the one deduced for D0 and D1 detectors. This result is compatible with a lower time of exposure to cosmic

\footnotetext{
2 Data corresponding to 111.4 days from a special set-up from October 2015 to February 2016 with only D0 and D2 detectors were used. In particular, profiting from the reduced cosmogenics in this period, D2 spectrum in coincidence with $1274.5 \mathrm{keV}$ depositions in D0 was analyzed. It is worth noting that the ${ }^{22} \mathrm{Na}$ initial activity in D0 deduced from the analogue analysis is in perfect agreement with the first estimate in [33].
} 
Table 3 Measured initial activities underground $\left(A_{0}\right)$ for the identified cosmogenic isotopes in $\mathrm{NaI}(\mathrm{Tl})$ crystals using ANAIS-25 data [33]. Half-lives of the products are also indicated [40]

\begin{tabular}{lll}
\hline Isotope & $T_{1 / 2}$ (days) & $A_{0}\left(\mathrm{~kg}^{-1} \mathrm{day}^{-1}\right)$ \\
\hline${ }^{126} \mathrm{I}$ & $12.93 \pm 0.05$ & $430 \pm 37$ \\
${ }^{125} \mathrm{I}$ & $59.407 \pm 0.009$ & $621.8 \pm 1.6$ \\
${ }^{127 m} \mathrm{Te}$ & $107 \pm 4$ & $32.1 \pm 0.8$ \\
${ }^{125 m} \mathrm{Te}$ & $57.40 \pm 0.15$ & $79.1 \pm 0.8$ \\
${ }^{123 m} \mathrm{Te}$ & $119.3 \pm 0.1$ & $100.8 \pm 0.8$ \\
${ }^{121 m} \mathrm{Te}$ & $154 \pm 7$ & $76.9 \pm 0.8$ \\
${ }^{121} \mathrm{Te}$ & $19.16 \pm 0.05$ & $110 \pm 12$ \\
${ }^{22} \mathrm{Na}$ & $(2.6029 \pm 0.0008)$ years & $159.7 \pm 4.9$ \\
\hline
\end{tabular}

rays, taking into account the ${ }^{22} \mathrm{Na}$ half-life, longer than that corresponding to I and Te products. As it will be discussed later, there are hints of the production of other isotopes like ${ }^{3} \mathrm{H},{ }^{109} \mathrm{Cd}$ and ${ }^{113} \mathrm{Sn}$ in the $\mathrm{NaI}(\mathrm{Tl})$ crystals, even if they could not be directly identified in the first analysis of cosmogenic activation presented in [33]. Apart from this, ${ }^{129}$ I can be present in the NaI crystals; it can be produced either as residual product of uranium spontaneous fission, or by cosmic rays reactions, having a broad range of activity values in iodine compounds depending on the ore origin. Due to its long lifetime and the difficulty to disentangle its signal from other emissions, the amount of ${ }^{129} \mathrm{I}$ in ANAIS-25 crystals could not be quantified. To take it into account in the background model its concentration was assumed to be the same as estimated by DAMA/LIBRA $\left({ }^{129} \mathrm{I} /{ }^{\text {nat }} \mathrm{I}=(1.7 \pm 0.1)\right.$ $10^{-13}$ ) [38], corresponding to an activity of $0.94 \mathrm{mBq} / \mathrm{kg}$.

\section{Background modeling}

The contribution of all the background sources described in Sect. 3 to the background levels of the ANAIS prototypes has been simulated by Monte Carlo using the Geant 4 package [41], as done in [18]. A detailed description of the set-ups was implemented including the lead shielding and detectors, considering $\mathrm{NaI}$ crystal, teflon wrapping, copper encapsulation with the Mylar window, silicone pads, quartz windows, PMTs, bases and copper enclosure; Fig. 2 shows the views of the Geant4 geometry for ANAIS-25 and ANAIS-37 setups. The Geant4 Radioactive Decay Module was used for simulating decays, after checking carefully the energy conservation in the decay of all the considered isotopes. The low energy models based on Livermore data libraries were considered for the physical processes of $\alpha, \beta$ and $\gamma$ emissions. Uniformly distributed bulk contamination in the components was assumed and activities (or derived upper limits) given in Tables 1, 2 and 3 considered. For each simulated event, defined considering an energy integration time of $1 \mu \mathrm{s}$, the energy deposited at each detector by different types of particles has been recorded separately in order to build afterwards the energy spectrum, filtering alpha deposits above $2.5 \mathrm{MeV}$ (as it can be made in real data by pulse shape analysis) and correcting each component with the corresponding Relative Scintillation Efficiency Factor. ${ }^{3}$ Production of scintillation at the $\mathrm{NaI}(\mathrm{Tl})$ crystals and the subsequent light collection have not been simulated here. Energy spectra at different conditions have been constructed to allow direct comparison to data obtained from detectors.

\section{Background contributions and comparison with data}

\subsection{ANAIS-25 detectors}

Figure 3 compares the energy spectrum summing all the simulated contributions described above with the measured data for ANAIS-25 detectors, considering anticoincidences or coincidences between the detectors. The last data taken at the ANAIS-25 set-up, from June 2014 to March 2015 corresponding to 231.55 days (live time) have been considered here; in these data most of the cosmogenic isotopes had decayed. A good agreement is obtained at high energy, but in the very low energy region some relevant contributions seem to be missing. Since upper limits on radionuclide activity have been used for several components, the background could be overestimated in some energy regions. The inclusion of cosmogenics was essential to reproduce in particular coincidence data.

The region from about 100 to $200 \mathrm{keV}$ was not well reproduced by simulations, mainly in the anticoincidence spectrum; the underestimation is partly solved when adding in the model the ${ }^{235} \mathrm{U}$ activity from the PMTs corresponding to the measured ${ }^{238} \mathrm{U}$ value and assuming the natural isotopic abundances of uranium, $\sim 7.2 \mathrm{mBq} / \mathrm{PMT}$. This value is very similar to that reported at [42]. In addition, the overestimation of the simulation around $92 \mathrm{keV}$ has been suppressed by reducing the ${ }^{238} \mathrm{U}$ upper limit for the copper vessel and quartz windows to that of ${ }^{226} \mathrm{Ra} .{ }^{4}$

In an attempt to find out the origin of the missing contributions at the very low energy region, different hypotheses have been analyzed:

- It was found that the inclusion in the model of an additional activity of $\sim 0.2 \mathrm{mBq} / \mathrm{kg}$ of ${ }^{3} \mathrm{H}$ in the NaI crystals significantly improves the agreement with data at low

\footnotetext{
3 A constant value of 0.6 has been taken as relative efficiency factor for alpha particles in the building of the electron equivalent energy spectra. Energy from nuclear recoils is neglected.

${ }^{4}$ Upper limits from gamma spectroscopy for the activity of isotopes at the upper part of the ${ }^{238} \mathrm{U}$ chain are typically much larger than those at the lower part starting on ${ }^{226} \mathrm{Ra}$ (as it can be seen in Table 1) because of the very low intensity of the gamma emissions at that chain segment.
} 

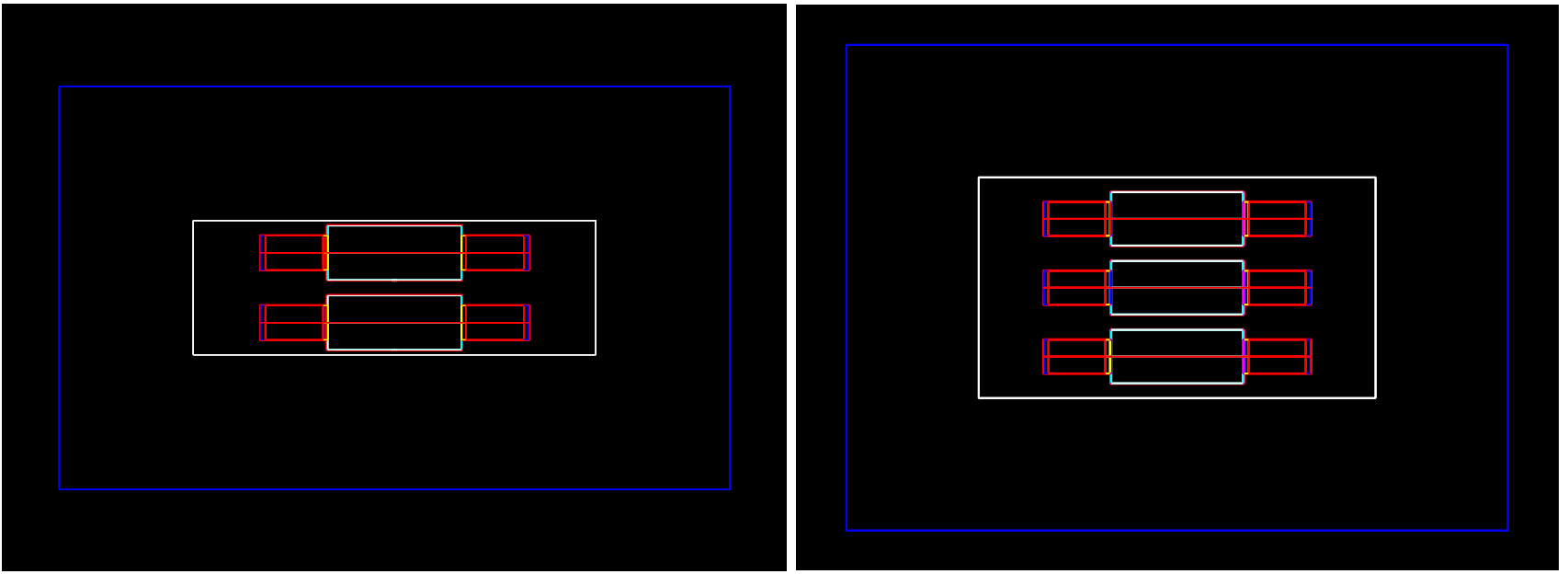

Fig. 2 Geometry of the ANAIS-25 (left) and ANAIS-37 (right) set-ups implemented in the Geant 4 simulations
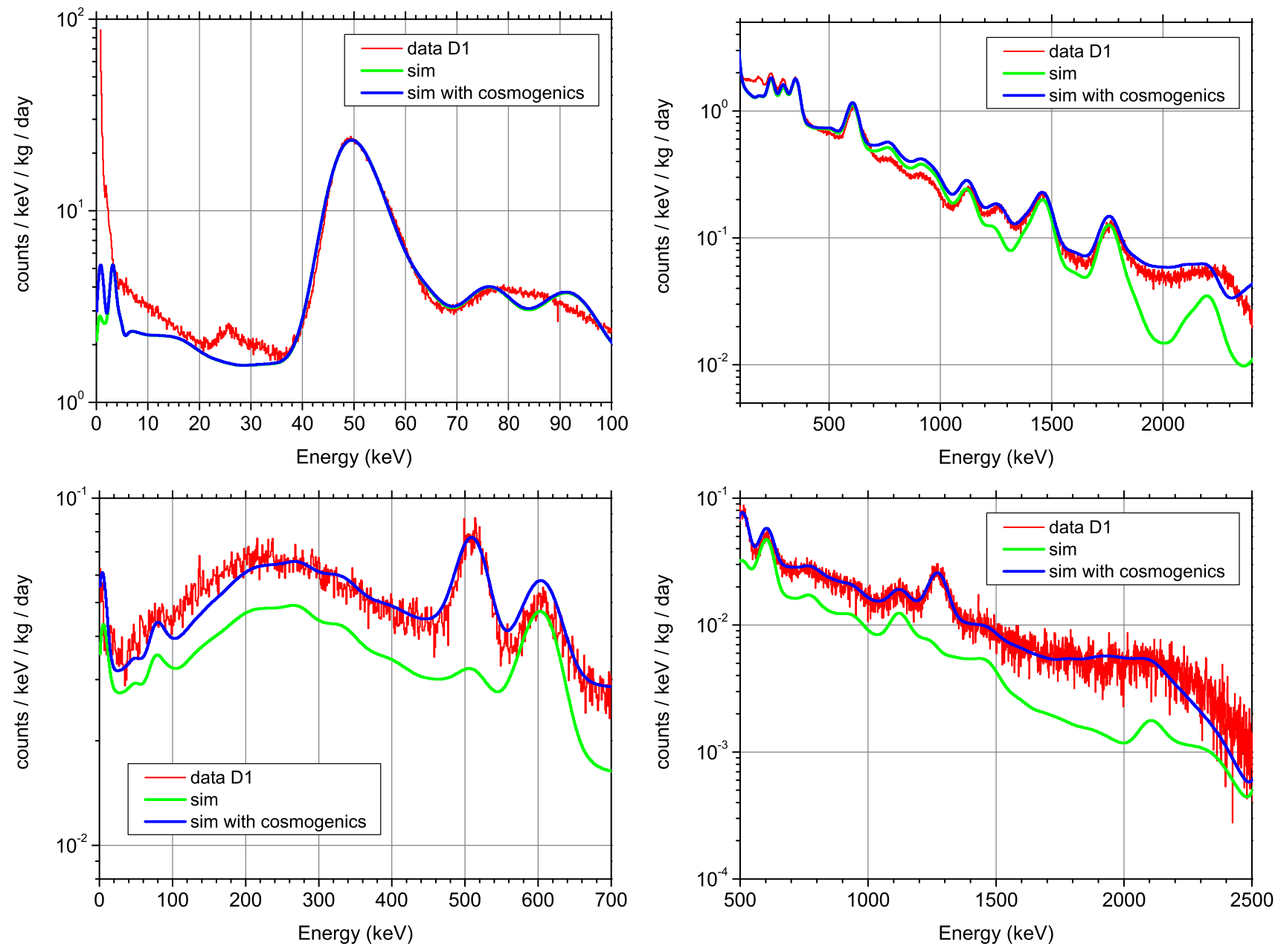

Fig. 3 Comparison of the energy spectra summing all the simulated contributions (before and after adding the cosmogenics) with the measured data for ANAIS-25 D1 detector considering anticoincidence (top)

and coincidence (bottom) data at low energy (left) and high energy $($ right $)$. Spectra for D0 detector are similar to those of D1 


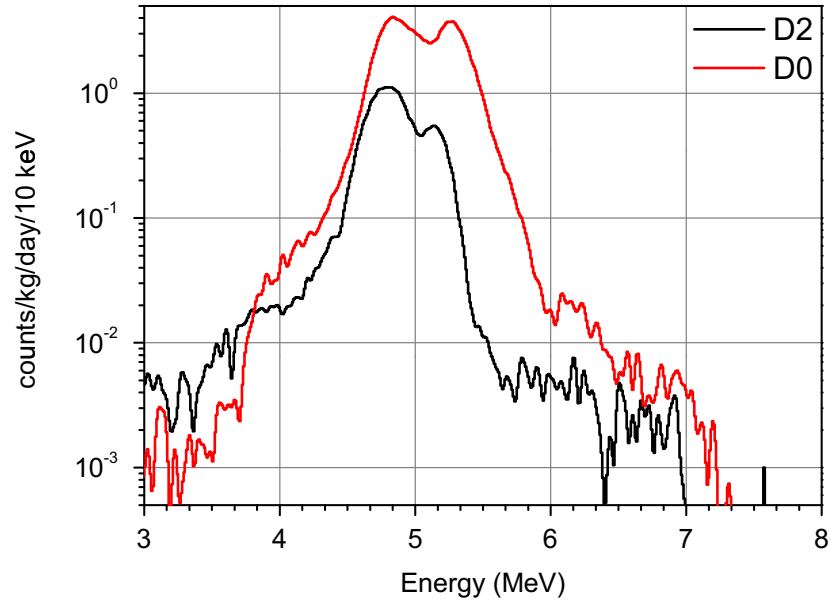

Fig. 4 Alpha region of the measured spectra for D0 (similar to that of D1) and D2 detectors. The peak from ${ }^{210}$ Po does not show the expected shape for a bulk contamination

energy. This value is about twice the upper limit set for DAMA/LIBRA crystals $(<0.09 \mathrm{mBq} / \mathrm{kg}$ [38]), but lower than the saturation activity which can be deduced from the production rates at sea level of ${ }^{3} \mathrm{H}$ in NaI calculated in [43] or [44], as described in [45].

- The unexplained peak around $25 \mathrm{keV}$ could be due to the cosmogenic production of ${ }^{109} \mathrm{Cd}$. This isotope decays by electron capture to the $88 \mathrm{keV}$ isomeric state of the daughter, having a half-life of 461.9 days, and therefore the peak may correspond to the binding energy of the Kshell of Ag. The observed peak can be reproduced using different exposure conditions and production rates of the order of the estimates made by convoluting production cross-sections with the cosmic neutron spectrum (see for instance [46], where a calculated rate of $4.8 \mathrm{~kg}^{-1} \mathrm{day}^{-1}$ is reported). It is worth noting that, being the case, an additional peak around $3.5 \mathrm{keV}$ ( $\mathrm{Ag} \mathrm{L}$-shell binding energy) is also expected, being 5.4 the ratio between $\mathrm{K}$ and L-shell EC probabilities [40].

- At the alpha region of the energy spectra measured for the detectors, the prominent peak due to the ${ }^{210} \mathrm{Po}$ emission (at the decay sequence of ${ }^{210} \mathrm{~Pb}$ ) does not show the pure structure expected from a crystal bulk ${ }^{210} \mathrm{~Pb}$ contamination (see Fig. 4) [24]. Therefore, the possibility of a surface deposition was carefully analyzed. Figure $5 \mathrm{com}-$ pares the low energy spectra simulated assuming ${ }^{210} \mathrm{~Pb}$ in bulk or in surface; since the surface contamination profile is unknown, different constant depths all around the crystal have been considered. ${ }^{5}$ The alpha emission is fully absorbed and only a small continuum appears at the left side of the peak for depths at or below $30 \mu \mathrm{m}$ in the

\footnotetext{
${ }^{5}$ Simulations with uniform emissions from the whole volume of a surface layer of the crystal have been also made; as an example, results for ${ }^{210} \mathrm{~Pb}$ contamination distributed in a $50-\mu \mathrm{m}$-thick surface layer are shown in Fig. 5 too.
}

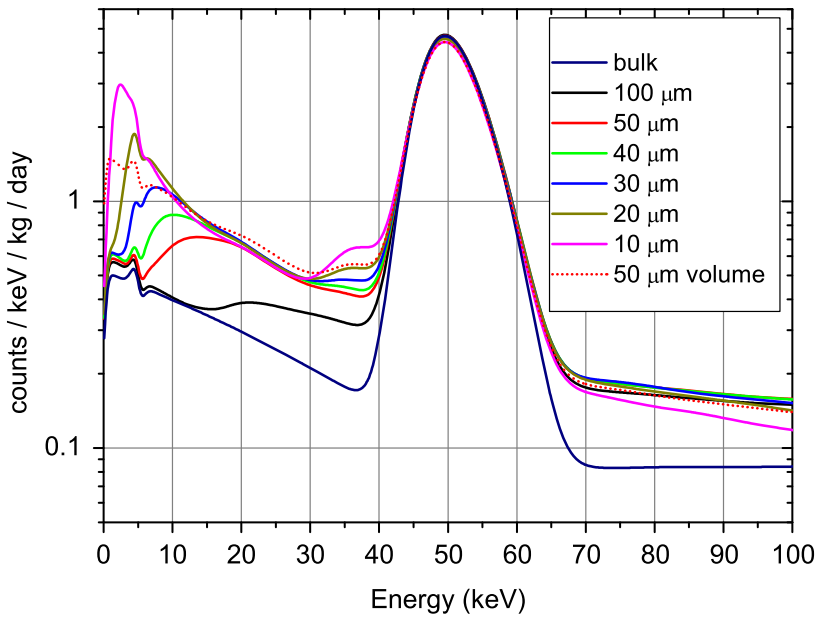

Fig. 5 Comparison of the low energy spectra simulated for ${ }^{210} \mathrm{~Pb}$ emissions from the crystal bulk or from the surface, considering different constant depths all around the crystal; results for uniform emissions from a $50-\mu \mathrm{m}$-thick layer are presented too. All simulations are normalized for an activity of $0.7 \mathrm{mBq} / \mathrm{kg}$

simulation. ${ }^{6}$ The need to reproduce the low energy region of the measured spectrum dominated by ${ }^{210} \mathrm{~Pb}$ emissions excludes surface contaminations at a very small depth. The best option to reproduce the whole range of data was found when considering half of the ${ }^{210} \mathrm{~Pb}$ content in bulk and the other half on surface from a constant depth of $100 \mu \mathrm{m}$; the bulk and surface proportion was fixed following the observed almost symmetric double structure of the ${ }^{210}$ Po peak for D0 and D1 detectors. This result has to be taken very cautiously, energy conversion into visible signal is assumed to be constant throughout the crystal in our simulation, but there should be a difference between energy depositions for alpha particles in two regions, as we are indirectly assuming when interpreting the double structure in the alpha peak as due to ${ }^{210} \mathrm{Po}$. These two regions could be surface and bulk. However, simple tests, including a similar reduced energy conversion for beta/gamma energy depositions having the same distribution than alpha contamination can be discarded, because it affects so much at the low energy events that it should have been clearly observed in the data. Nevertheless, we cannot discard some spatial dependence of the energy conversion that could affect differently alpha particles than beta/gammas and then, it could affect too the energy depositions at the lowest energies (below $10 \mathrm{keV}$ and not in the $50 \mathrm{keV}$ energy scale).

Figure 6 compares again the measured ANAIS-25 spectra with the simulated ones including all these hypotheses,

\footnotetext{
6 The range of a $5-\mathrm{MeV}$ alpha particle in $\mathrm{NaI}$ is $29 \mu \mathrm{m}$, following NIST data [47].
} 

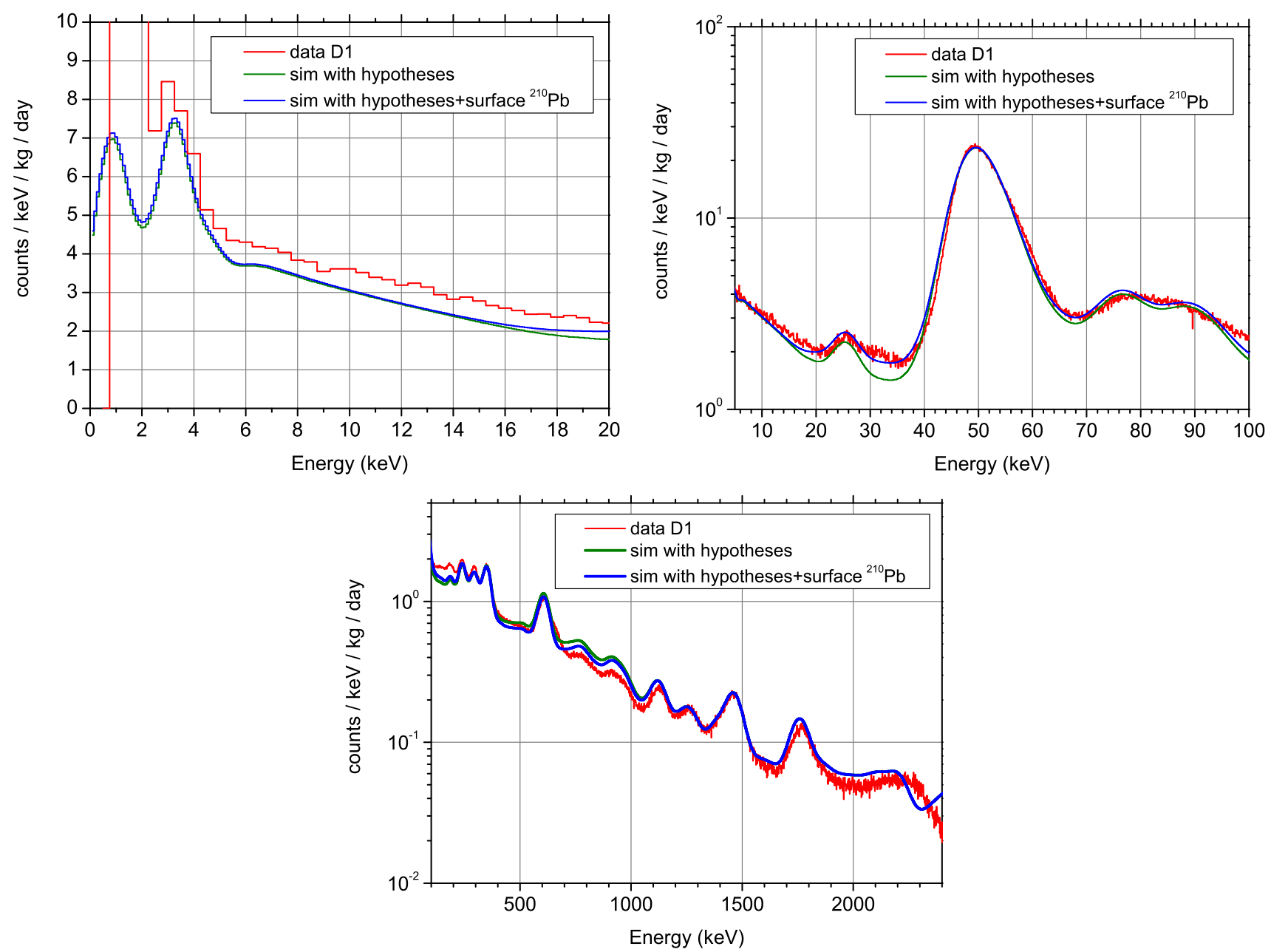

Fig. 6 Effect of the consideration of some background hypotheses in the spectra of ANAIS-25 detectors at all energy ranges for anticoincidence data (see text). The inclusion of some reduced ${ }^{238} \mathrm{U}$ upper limits, ${ }^{235} \mathrm{U}$ at PMTs and ${ }^{3} \mathrm{H}$ and ${ }^{109} \mathrm{Cd}$ at crystals has been considered (green

for anticoincidence data. The model still gives an overestimation from 0.6 to $1 \mathrm{MeV}$, since upper limits have been considered for several contaminations, and an underestimation from 100 to $200 \mathrm{keV}$. The inclusion of both ${ }^{3} \mathrm{H}$ and ${ }^{109} \mathrm{Cd}$ contributions significantly improves the agreement in the lowest energy region. The additional inclusion of ${ }^{210} \mathrm{~Pb}$ partly on surface helps to reproduce the region from 30 to $40 \mathrm{keV}$.

Figure 7, left summarizes the different contributions from the explained background model of ANAIS-25 detectors, for anticoincidence data, to the rate in the region from 1 to 6 keVee. The energy spectra expected from different background sources in the very low energy region for anticoincidence data are plotted in Fig. 7, right, together with the sum of all contributions. In the RoI ${ }^{210} \mathrm{~Pb}$ and ${ }^{3} \mathrm{H}$ continua and ${ }^{40} \mathrm{~K},{ }^{22} \mathrm{Na}$ and ${ }^{109} \mathrm{Cd}$ peaks are the most significant contributions. line); the additional assumption of half of the ${ }^{210} \mathrm{~Pb}$ emission from a depth of $100 \mu \mathrm{m}$ on the crystal surface is separately shown (blue line). The considered hypotheses allow to significantly improve the overall agreement with measured data

\subsection{D2 module at ANAIS-37}

Figure 8 compares the energy spectrum measured for D2 detector at the ANAIS-37 set-up with the corresponding simulation. The data taken with this set-up from May to September 2015 for 89.5 days have been considered here; in these data the cosmogenic activation has significantly, but not completely, decayed. For the simulation, the input activities given in Tables 1 and 2 for D2 have been assumed. The initial activity of I and Te products cosmogenically induced in the NaI crystal has been taken the same determined for D0 and D1 detectors, although previous exposure history of the detectors is not the same and some differences could be expected in other longer half-life isotopes, for I and Te products saturation should have been reached nonetheless. For ${ }^{22} \mathrm{Na}$, the initial activity quantified specifically for D2 detector (see Sect. 3) has been considered. The overall agreement 


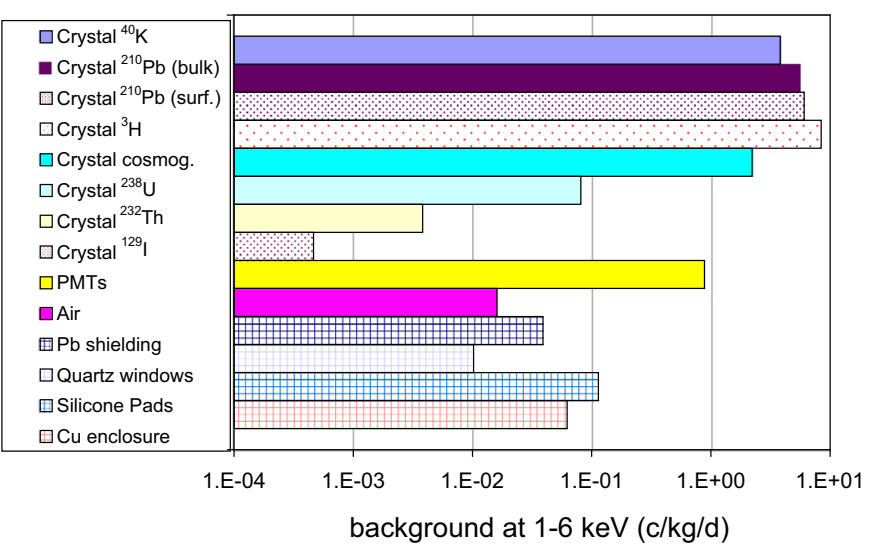

Fig. 7 ANAIS-25 background model: expected rates from different background sources in the region of 1-6 keVee (left) and the corresponding spectra at the very low energy region (right). In the left plot,

between data and simulation in Fig. 8 is quite satisfactory except for some energy regions. As for ANAIS-25 detectors, ${ }^{210} \mathrm{~Pb}$ emissions at low energy are well reproduced assuming the activity deduced from the alpha rate. At medium energies around $1 \mathrm{MeV}$, the simulation is clearly overestimated; this could be due again to the fact that upper limits on radionuclide activity have been used for several components.

The observed peak around $185 \mathrm{keV}$ in D2 data and not reproduced by simulation (as well as the underestimation of that at $\sim 145 \mathrm{keV}$ ) could be justified by the content of ${ }^{235} \mathrm{U}$ from the PMTs, also considered for D0 and D1 detectors (see Sect. 5.1). Again as for ANAIS-25, the overestimation of the simulation around $92 \mathrm{keV}$ can be partially suppressed by reducing the ${ }^{238} \mathrm{U}$ upper limit for the copper vessel and quartz windows to that of ${ }^{226} \mathrm{Ra}$. Some plausible hypotheses have been analyzed to find an explanation to the relevant discrepancies in the low energy region:

- The prominent line registered at $\sim 28 \mathrm{keV}$ is not explained by the cosmogenic isotopes identified in ANAIS-25 data. ${ }^{113} \mathrm{Sn}$, having a half-life of 115.1 days and decaying by electron capture mainly to a $391.7 \mathrm{keV}$ isomeric state of the daughter, could justify this spectral feature as binding energy of In K-shell and activation of the isotope is possible. As for ${ }^{109} \mathrm{Cd}$, the observed peak can be reproduced using different exposure conditions and production rates of the order of the estimates made by convoluting production cross-sections with the cosmic neutron spectrum (at [46], a calculated rate of $9 \mathrm{~kg}^{-1} \mathrm{day}^{-1}$ is reported and a measured value of $16 \mathrm{~kg}^{-1} \mathrm{day}^{-1}$ presented). A peak around $4 \mathrm{keV}$ is expected from ${ }^{113} \mathrm{Sn}$ as the binding energy of the L-shell of In (the ratio between the probabilities of electron capture for $\mathrm{K}$ and $\mathrm{L}$ shells is 7.4 for

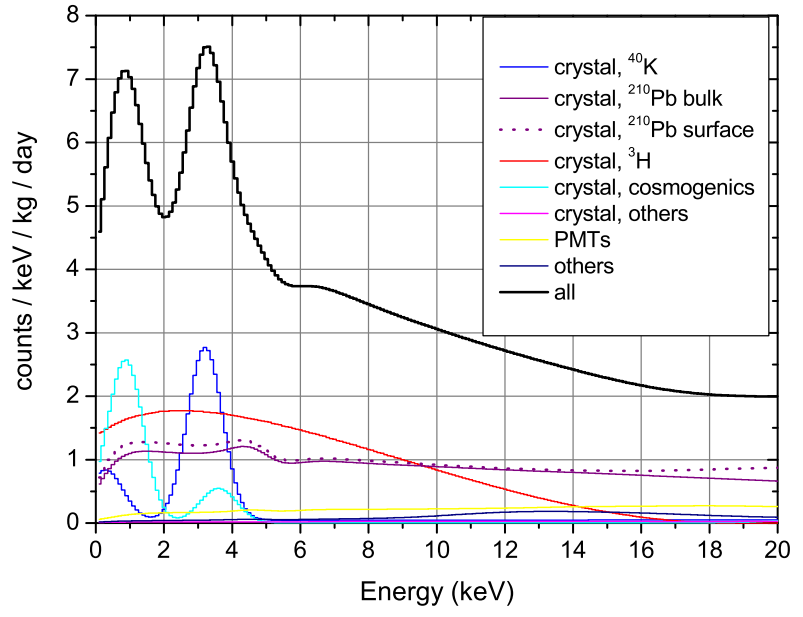

some contributions have been estimated from a directly quantified activity (filled bars) but others from upper limits (plaid bars) or hypothesized activities (dotted bars)

decay to the isomeric state [40]), but since the half-life of ${ }^{113} \mathrm{Sn}$ is not too large, it should not be a problem in the long term. ${ }^{113} \mathrm{Sn}$ induced in D0/D1 detectors should have decayed in the data analyzed here. No hint of the presence of ${ }^{109} \mathrm{Cd}$, as seen in ANAIS-25 detectors, can be observed for the moment in D2 data; as for ${ }^{22} \mathrm{Na}$, the initial activity of this isotope, having a longer half-life than ${ }^{113} \mathrm{Sn}$, could be lower than in D0 and D1 due to the different time spent at Colorado.

- The spectrum shape and rate observed below $20 \mathrm{keV}$ in D2 data are not completely reproduced by simulation. Several possibilities have been explored:

- Considering the amount of ${ }^{3} \mathrm{H}$ deduced for ANAIS25 detector, the spectral shape of its beta emission does not fully explain the observed background below $20 \mathrm{keV}$; therefore, its content has been fixed just to the upper limit set by DAMA/ LIBRA $(0.09 \mathrm{mBq} / \mathrm{kg}$ [38]). Due to the shorter exposure to cosmic rays in Colorado of D2 in comparison to D0 and D1, a lower ${ }^{3} \mathrm{H}$ activity is expected.

- The possibility of ${ }^{210} \mathrm{~Pb}$ emissions on the crystal surface instead of in bulk has been deeply studied too. As shown in Fig. 4, the double structure of the ${ }^{210} \mathrm{Po}$ peak observed at the alpha region of the energy spectra of $\mathrm{D} 2$ detector is more asymmetric than that measured for D0/D1; according to this peak structure, a fourth of the ${ }^{210} \mathrm{~Pb}$ contamination has been considered in the crystal bulk and the rest on surface. For D2 detector, and considering all the possibilities analyzed (see Fig. 5 and Sect. 5.1 for more details), the best option to reproduce the low energy region of the spectra dominated by ${ }^{210} \mathrm{~Pb}$ emissions was a surface contamination from a constant depth of $30 \mu \mathrm{m}$. 

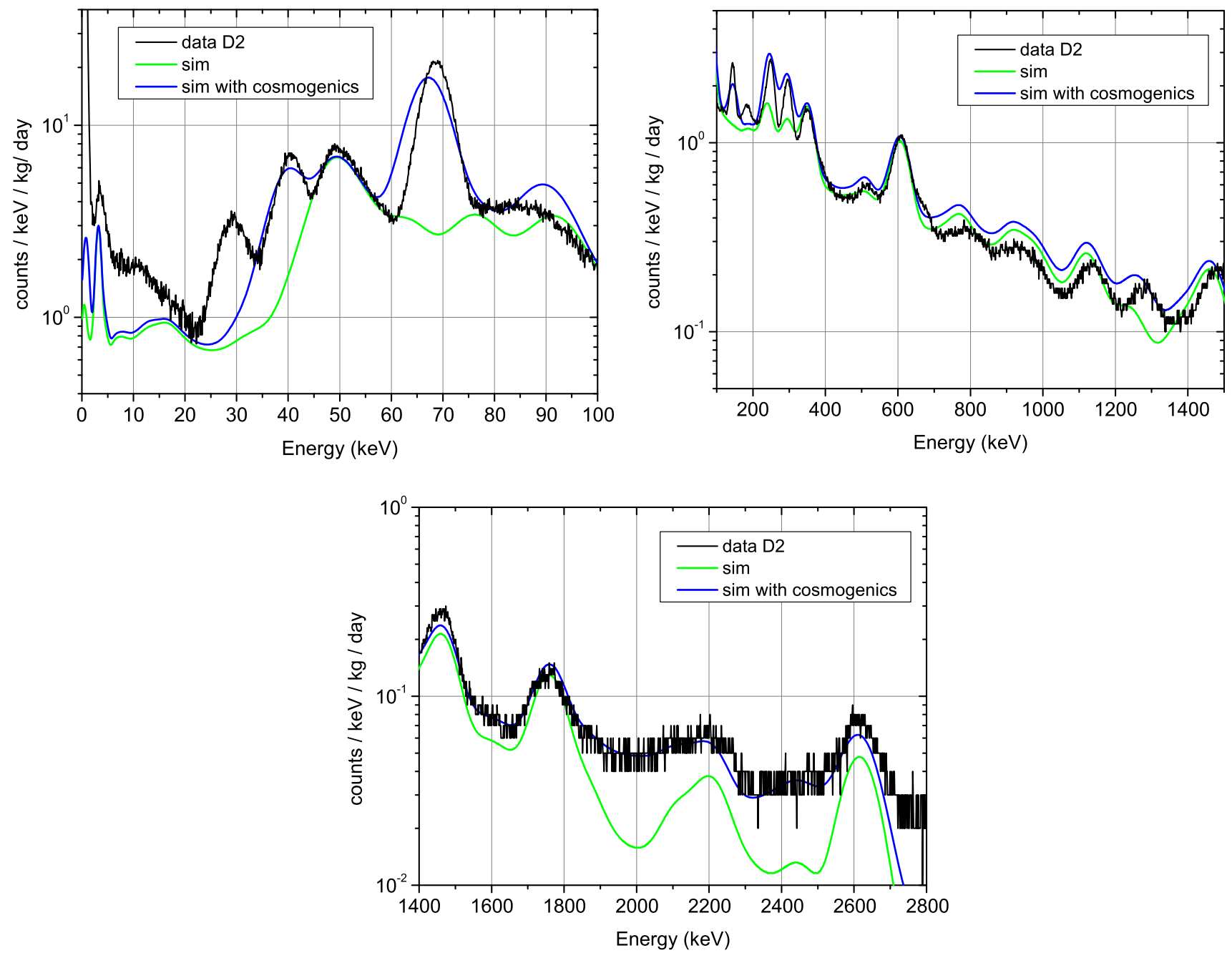

Fig. 8 Comparison of the energy spectrum measured for D2 detector at the ANAIS-37 set-up with the corresponding simulation summing all contributions (before and after adding the cosmogenics) at low, medium

Figure 9 compares the measured D2 spectra with the simulated ones taking into consideration all the described hypotheses, which significantly improve the agreement. It is remarkable that the inclusion of ${ }^{3} \mathrm{H}$ in the model is necessary, since only surface and bulk ${ }^{210} \mathrm{~Pb}$ emissions cannot reproduce the registered spectrum. The higher continuum level below $40 \mathrm{keV}$ produced by the ${ }^{210} \mathrm{~Pb}$ surface emission in comparison with the bulk contamination helps to make the model be closer to the measured data.

Figure 10, left summarizes the different contributions from the proposed background model of D2 at the ANAIS37 set-up, for anticoincidence data, to the rate in the region from 1 to $6 \mathrm{keVee}$. The energy spectra expected from different background sources in the very low energy region for anticoincidence data are plotted in Fig. 10, right, together with the sum of all contributions. In this RoI it has been verified that ${ }^{210} \mathrm{~Pb}$ contribution has been significantly reduced and high energy regions. Anticoincidence data are shown in the low energy region

in comparison to D0 and D1; but it is worth pointing out that emissions from surface make a larger contribution than from bulk. Peaks from ${ }^{113} \mathrm{Sn}$ and, to a lesser extent, ${ }^{22} \mathrm{Na}$ will be decreasing in the next future.

As conclusion, and according to our background model, surface contamination in ${ }^{210} \mathrm{~Pb}$ is needed to reproduce low energy spectra of D0/D1 and D2 modules, even though in a different amount and in different depth profile. Although some important assumptions are required to estimate the contribution of surface contaminants to the energy spectra, and then, the contaminations depths for ${ }^{210} \mathrm{~Pb}$ derived from our analysis should not be firmly taken as stated, we can conclude that a fraction of the total contamination in ${ }^{210} \mathrm{~Pb}$ could be due to the treatment of the surfaces while the building of D0/D1 and D2 modules. In [48-50], diffusion lengths for radon-induced surface contamination which could happen due to exposure to air during assembly phase and storage 

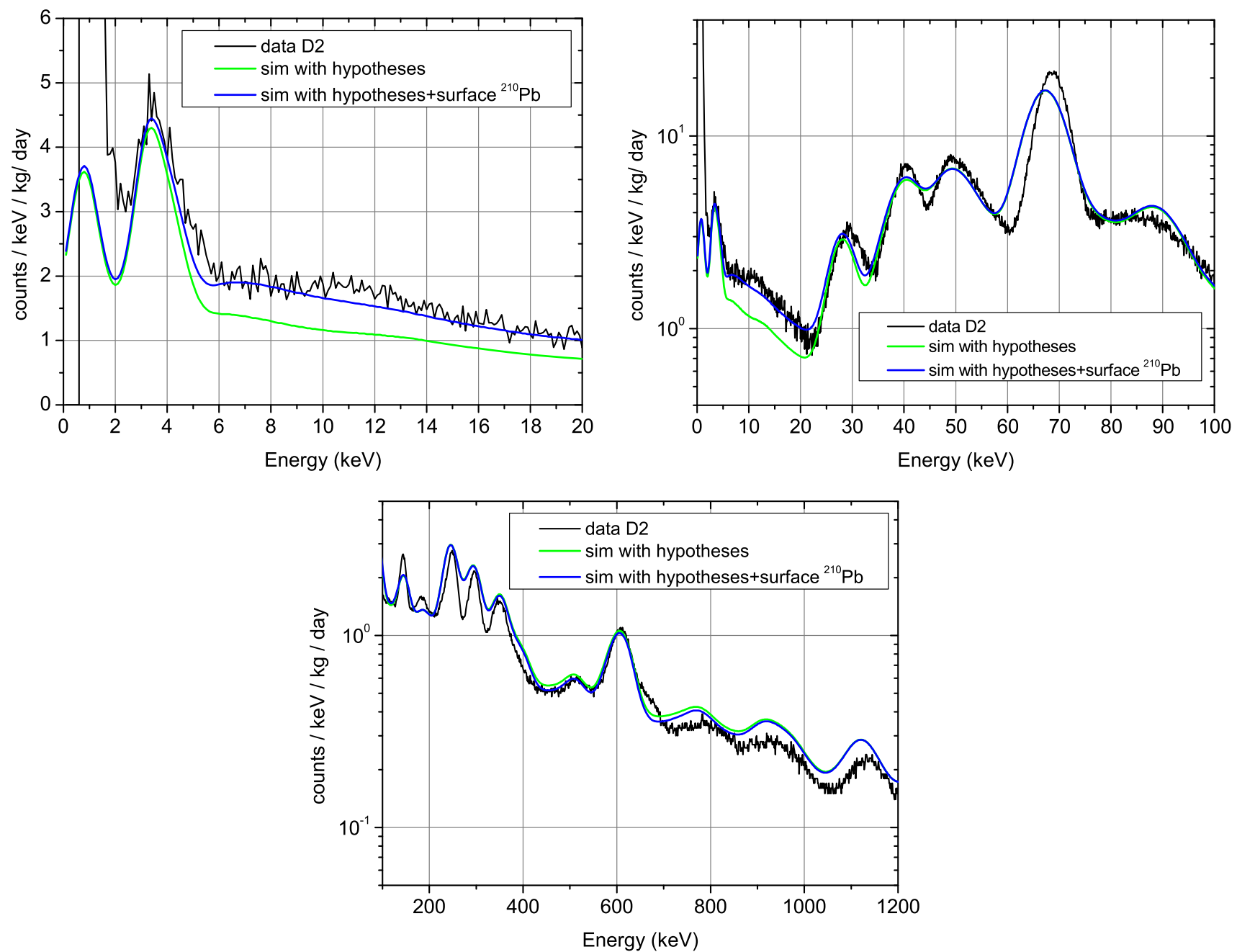

Fig. 9 Effect of the consideration of the plausible analyzed background hypotheses (see text) in the spectra of D2 detector at different energy ranges (at the highest energies the hypotheses have no effect). The inclusion of some reduced ${ }^{238} \mathrm{U}$ upper limits, ${ }^{235} \mathrm{U}$ at PMTs and ${ }^{3} \mathrm{H}$ and ${ }^{113} \mathrm{Sn}$ at crystal has been considered (green line); the additional assumption of part of the ${ }^{210} \mathrm{~Pb}$ emission from a depth of $30 \mu \mathrm{m}$ on the crystal surface is separately shown (blue line). The considered hypotheses are essential to improve the overall agreement with measured data of detectors are estimated below $1 \mu \mathrm{m}$, depending on radon concentration, exposure time and features of material surface; the required depths deduced for ANAIS crystals cannot then be explained by diffusion from radon surface deposition, but they would point to other mechanisms allowing the contamination from radon (or even directly ${ }^{210} \mathrm{~Pb}$ ) at the production phase of the crystals or at the treatment of surfaces. This issue is being further investigated in collaboration with AS company.

\section{Background projections}

ANAIS in collaboration with AS is strongly pursuing an improvement of the radiopurity of the subsequent $12.5 \mathrm{~kg}$ modules, trying to focus in further reduction of the powder ${ }^{40} \mathrm{~K}$ content, in reducing ${ }^{22} \mathrm{Na}$ activation by using convenient shieldings while storing at surface, and avoiding ${ }^{210} \mathrm{~Pb}$ contamination. In parallel, background rejection power of coincidences has been analyzed in different experimental scenarios for ANAIS: present configuration with $3 \times 3$ modules $(112.5 \mathrm{~kg})$ and also an enlarged experiment using $4 \times 5$ modules $(250 \mathrm{~kg})$. An additional Liquid Scintillator Veto (LSV) surrounding the $\mathrm{NaI}(\mathrm{Tl})$ detectors has been also considered (see Fig. 11).

Shape and size of ANAIS-37 modules have been assumed in all this analysis. For the LSV system, Linear AlkylBenzene (LAB) has been considered as scintillator medium inside a cylindrical stainless steel vessel read by five $8^{\prime \prime}$ Hamamatsu R5912 PMTs at each side; a scintillator thickness of $60 \mathrm{~cm}$ 


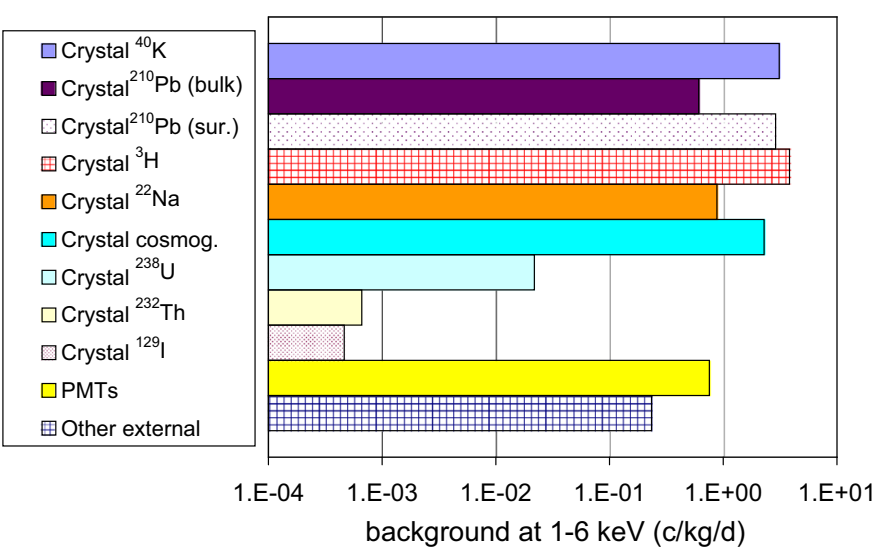

Fig. 10 Background model for D2 at the ANAIS-37 set-up: expected rates from different background sources in the region of $1-6 \mathrm{keVee}(l e f t)$ and the corresponding spectra at the very low energy region (right). In the left plot, some contributions have been estimated from a directly

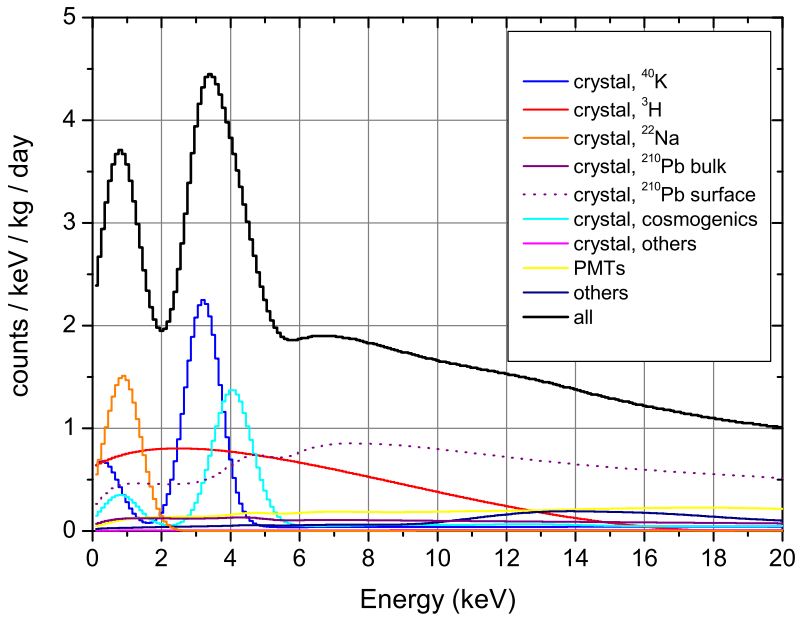

quantified activity (filled bars) but others from upper limits (plaid bars) or hypothesized activities (dotted bars). Contribution from cosmogenic isotopes has been evaluated for the period corresponding to the real data taking
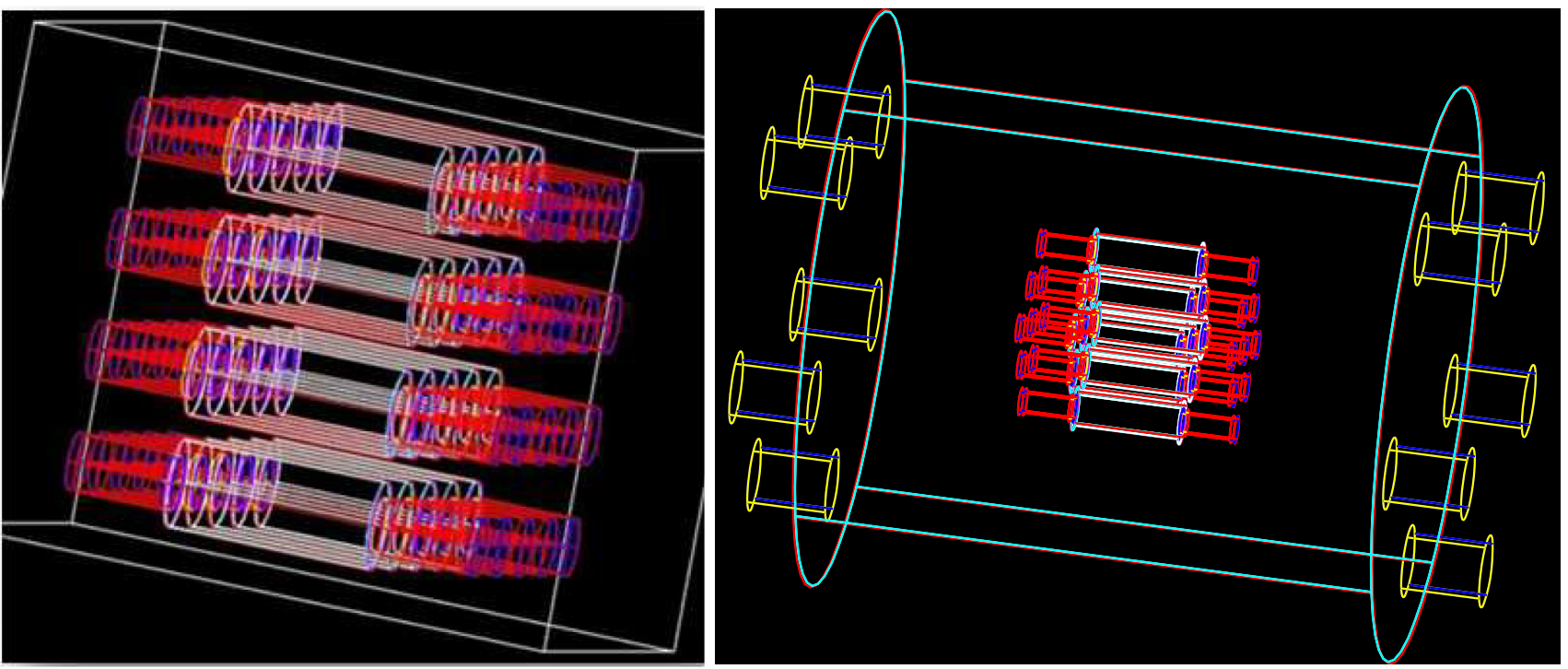

Fig. 11 Geometry of some possible full set-ups for ANAIS, as implemented in Geant 4 simulations: with $4 \times 5 \mathrm{NaI}(\mathrm{Tl})$ modules $(l e f t)$ and with $3 \times 3 \mathrm{NaI}(\mathrm{Tl})$ detectors inside a liquid scintillator veto (right)

at top, bottom and side of the cylindrical vessel has been assumed (corresponding to a total mass of $3.8 \mathrm{t}$ ). For all the full ANAIS set-ups simulated, measured primordial activities from PMTs and crystals (assuming $0.7 \mathrm{mBq} / \mathrm{kg}$ of bulk ${ }^{210} \mathrm{~Pb}$ and $1.25 \mathrm{mBq} / \mathrm{kg}$ of ${ }^{40} \mathrm{~K}$ ) have been included. The cosmogenic initial activity of ${ }^{22} \mathrm{Na}$ and possible tritium contribution have been taken into account too, at the levels deduced for D2. Concerning the activity of the LSV system itself, values from the literature have been considered: from NEXT for stainless steel [51], SNOlab for PMTs [52] and JUNO for LAB scintillator [53]. According to the obtained results for the assumed geometry and activities, the contribution to the background levels of the LSV components is negligible (two orders of magnitude lower) in comparison to that of the $\mathrm{NaI}(\mathrm{Tl})$ crystals; for this reason, the $3 \times 3$ modules set-up without a LSV system has not been independently simulated.

Figure 12 compares the energy spectra at the very low energy region expected from all the considered background sources in different situations, to illustrate the effect of anticoincidence between crystals and also with the LSV. Spectra have been evaluated for the $3 \times 3$ detectors set-up considering anticoincidence only between crystals, also with an ideal LSV and with a LSV having a $500 \mathrm{keV}$ energy threshold. Spectra obtained in anticoincidence at the $4 \times 5$ modules 


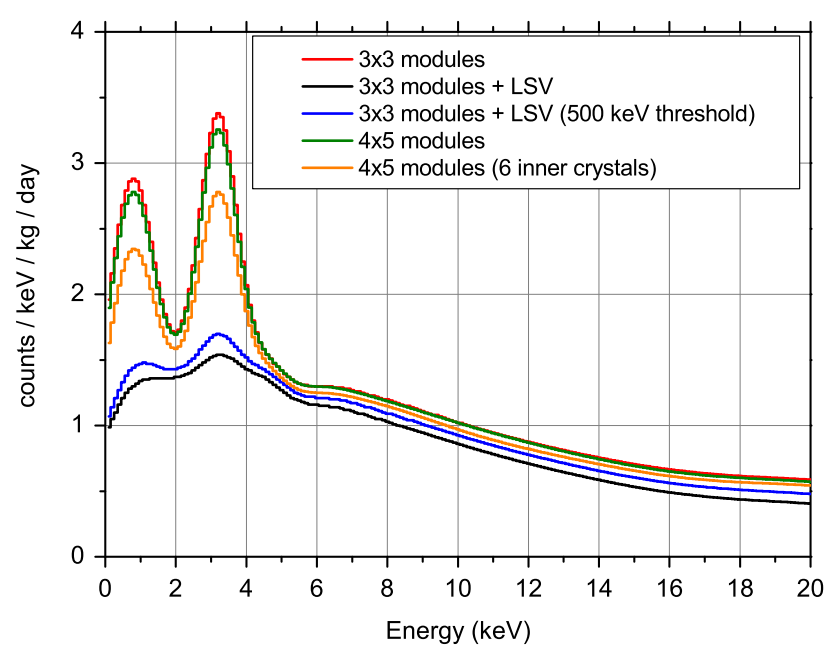

Fig. 12 Energy spectra at the very low energy region expected from all the considered background sources in different set-ups and anticoincidence conditions (see text). The presently achieved radiopurity of D2 module has been assumed for all the modules

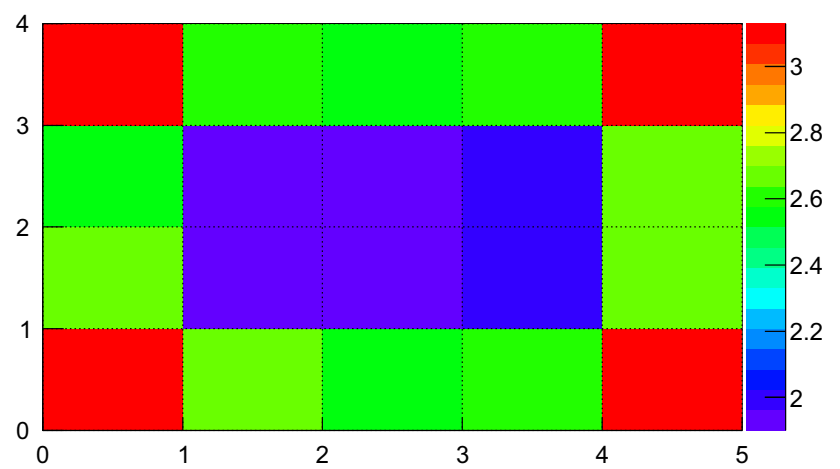

Fig. 13 Distribution of the background level (expressed in $\mathrm{c} / \mathrm{kg}^{-1} / \mathrm{day}^{-1}$ ) registered in the $1-6 \mathrm{keVee}$ region in anticoincidence at each crystal over a matrix of 4 (vertical) by 5 (horizontal) detectors, for ${ }^{40} \mathrm{~K}$ emissions from the $\mathrm{NaI}(\mathrm{Tl})$ crystals. Similar plots are obtained for ${ }^{22} \mathrm{Na}$ also from crystals and for PMTs emissions; they indicate that the analysis of only the six inner modules could be advantageous

set-up are also presented; in this configuration it has been observed that considering only the six inner crystals, and not all of the twenty detectors, produces a lower background level at the RoI (see Fig. 13) and results in these two conditions are shown. As expected, the reduction by anticoincidence is more modest in a $3 \times 3$ matrix than in a $4 \times 5$ configuration. But the implementation of a LSV system at the $3 \times 3$ matrix set-up is more effective that the increase of NaI modules, for fixed radiopurity levels. Concerning the contribution at the RoI from ${ }^{210} \mathrm{~Pb}$ in crystals, it is worth noting that it could be higher in case of a surface contamination (as shown in Fig. 5); for instance, if all the ${ }^{210} \mathrm{~Pb}$ was on surface at a constant depth of $30 \mu \mathrm{m}$, the increase in the rate in the 1-6 keVee region in comparison to a pure bulk contamination would be of 1.6 .
Table 4 summarizes the reduction factors (R.F.) obtained in the considered situations at the 1-6 keVee energy region, for all the contributions altogether and separately for some background sources, those for which high reduction is expected: ${ }^{40} \mathrm{~K}$ and ${ }^{22} \mathrm{Na}$ at crystals and PMT contaminations. The R.F. is computed from the ratio between the rates in the 1-6 keVee after coincidence events rejection and before any rejection and therefore corresponds to the percentage of remaining background. The veto by the LSV is very effective for the ${ }^{40} \mathrm{~K}$ and ${ }^{22} \mathrm{Na}$ peaks, but since it is useless for the other relevant background sources at the RoI $\left({ }^{3} \mathrm{H}\right.$ and ${ }^{210} \mathrm{~Pb}$ ) the estimated overall R.F. is in the end of $55.6 \%$ for the ideal LSV and $59.1 \%$ assuming a $500 \mathrm{keV}$ threshold (see Table 4). For the assumed background model, about $70 \%$ of the background obtained for the $3 \times 3$ crystals configuration in anticoincidence remains after the vetoing effect of the LSV.

\section{Conclusions}

The background models constructed for D0 and D1 detectors at the ANAIS-25 set-up and for D2 module at ANAIS37 provide a good description of measured data at all energy ranges and at different analysis conditions (coincidence/anticoincidence). The measured activity in external components and in crystal, including cosmogenic products and quantified combining different analysis techniques, roughly explains the observed background. But the inclusion of some additional hypotheses, like the presence of cosmogenic isotopes which cannot be directly quantified or partial crystal surface ${ }^{210} \mathrm{~Pb}$ contamination, significantly improves the agreement between model and real data.

Reduction factors for the background at the very low energy region thanks to the rejection of coincident events have been computed for the full ANAIS set-up and for several hypothetical scenarios, like a matrix of $\mathrm{NaI}(\mathrm{Tl})$ crystals corresponding to a total mass of $250 \mathrm{~kg}$, or the use of a liquid scintillator veto in the $3 \times 3$ modules configuration.

The measured background in D2 is already about 2 counts/(keV kg day) at $\sim 6 \mathrm{keVee}$ but reduction is still possible thanks to the increase of the background rejection power in a detector matrix set-up and an improved control of radiopurity. Thanks to the already achieved reduction in D2 detector of ${ }^{210} \mathrm{~Pb}$ activity, its contribution in the RoI is at some tenths of count/(keV kg day). That of PMTs and other external components is below 0.2 counts/(keV kg day). ${ }^{40} \mathrm{~K}$ and ${ }^{22} \mathrm{Na}$ peaks can be significantly reduced by anticoincidence operation of the full experiment. With the radiopurity levels achieved in $\mathrm{D} 2$ detector, a background rate below 2 counts/( $\mathrm{keV} \mathrm{kg}$ day) above $4 \mathrm{keVee}$ is expected for a $3 \times 3$ matrix of crystals amounting to $112.5 \mathrm{~kg}$ of mass; with these activity levels of D2, the use of a LSV is clearly a better alter- 
Table 4 Reduction factor (R.F., defined as \% of background remaining after anticoincidence in the 1-6 keVee region) computed for several set-ups and applying anticoincidence rejection only between crystals or also with the LSV (see text). It has been evaluated separately for some background sources and for the overall background in different experimental scenarios

\begin{tabular}{|c|c|c|c|c|}
\hline Set-up & $\begin{array}{l}\text { R.F. }(\%) \\
{ }^{40} \mathrm{~K} \text { from crystals }\end{array}$ & $\begin{array}{l}\text { R.F. }(\%) \\
{ }^{22} \mathrm{Na} \text { from crystals }\end{array}$ & $\begin{array}{l}\text { R.F. (\%) } \\
\text { PMTs }\end{array}$ & $\begin{array}{l}\text { R.F. }(\%) \\
\text { Total }\end{array}$ \\
\hline $3 \times 3$ modules & 69.0 & 62.4 & 62.3 & 83.7 \\
\hline $3 \times 3$ modules $+\mathrm{LSV}$ & 11.9 & 1.2 & 7.3 & 55.6 \\
\hline $3 \times 3$ modules $+\mathrm{LSV}(500 \mathrm{keV}$ threshold $)$ & 15.5 & 5.7 & 29.3 & 59.1 \\
\hline $4 \times 5$ modules & 62.7 & 55.1 & 45.9 & 77.9 \\
\hline $4 \times 5$ modules ( 6 inner crystals) & 48.6 & 39.8 & 32.5 & 70.3 \\
\hline
\end{tabular}

native than the increase of the total mass up to $250 \mathrm{~kg}$, and the background rate is reduced below 1.5 counts/( $\mathrm{keV} \mathrm{kg}$ day) at the whole RoI. In this situation, the fully-absorbed emission from ${ }^{3} \mathrm{H}$ could become the main contribution in the region of interest; therefore, a shielding against cosmogenic activation has been procured for the production of the new ANAIS $\mathrm{NaI}(\mathrm{Tl})$ crystals. Additionally, a further reduction of ${ }^{210} \mathrm{~Pb}$ and ${ }^{40} \mathrm{~K}$ in Alpha Spectra detectors of WIMPScint-III grade can be foreseen thanks to improved purification and surface machining protocols.

Acknowledgments This work has been supported by the Spanish Ministerio de Economía y Competitividad and the European Regional Development Fund (MINECO-FEDER) (FPA2011-23749 and FPA2014-55986-P), the Consolider-Ingenio 2010 Programme under Grants MULTIDARK CSD2009-00064 and CPAN CSD200700042, and the Gobierno de Aragón and the European Social Fund (Group in Nuclear and Astroparticle Physics). P. Villar is supported by the MINECO Subprograma de Formación de Personal Investigador. We also acknowledge LSC and GIFNA staff for their support.

Open Access This article is distributed under the terms of the Creative Commons Attribution 4.0 International License (http://creativecomm ons.org/licenses/by/4.0/), which permits unrestricted use, distribution, and reproduction in any medium, provided you give appropriate credit to the original author(s) and the source, provide a link to the Creative Commons license, and indicate if changes were made. Funded by SCOAP ${ }^{3}$.

\section{References}

1. K. Fushimi et al., Limits on the annual modulation of WIMPs nucleus scattering with large-volume $\mathrm{NaI}(\mathrm{Tl})$ scintillators. Astropart. Phys. 12, 185 (1999)

2. M.L. Sarsa et al., Results of a search for annual modulation of WIMP signals. Phys. Rev. D 56, 1856 (1997)

3. R. Bernabei et al., Performances of the $100 \mathrm{~kg} \mathrm{NaI}$ (Tl) set-up of the DAMA experiment at Gran Sasso. Riv. Nuovo Cim. A 112, 545 (1999)

4. G. Gerbier et al., Pulse shape discrimination with $\mathrm{NaI}(\mathrm{Tl})$ and results from a WIMP search at the Laboratoire Souterrain de Modane. Astropart. Phys. 11, 287 (1999)

5. G.J. Alner et al., Limits on WIMP cross-sections from the NAIAD experiment at the Boulby Underground Laboratory. Phys. Lett. B 616, 17 (2005)
6. R. Bernabei et al., Final model independent result of DAMA/LIBRA-phase1. Eur. Phys. J. C 73, 2648 (2013)

7. R. Agnese et al., New Results from the Search for Low-Mass Weakly Interacting Massive Particles with the CDMS Low Ionization Threshold Experiment. Phys. Rev. Lett. 116, 071301 (2016)

8. CRESST Collaboration, G. Angloher et al., CRESST results on light dark matter particles with a low-threshold CRESST-II detector. Eur. Phys. J. C 76, 25 (2016)

9. EDELWEISS Collaboration, E. Armengaud et al., Constraints on low-mass WIMPs from the EDELWEISS-III dark matter search. JCAP 05, 019 (2016)

10. H.S. Lee et al., A search for low-mass dark matter with $\mathrm{CsI}(\mathrm{Tl})$ crystal detectors. Phys. Rev. D 90, 052006 (2014)

11. LUX Collaboration, D. Akerib et al., First results from the LUX dark matter experiment at the Sanford underground research facility. Phys. Rev. Lett. 112, 091303 (2014)

12. C. Amole et al., Dark matter search results from the PICO- $60 \mathrm{CF}_{3} \mathrm{I}$ bubble chamber. Phys. Rev. D 93, 052014 (2016)

13. XENON Collaboration, E. Aprile et al., XENON search for event rate modulation in XENON100 electronic recoil data. Phys. Rev. Lett. 115, 091302 (2015)

14. J. Amaré et al., Status of the ANAIS dark matter project at the Canfranc Underground Laboratory. J. Phys. Conf. Ser. 718, 042052 (2016)

15. J. Cherwinka et al., First data from DM-Ice17. Phys. Rev. D 90, 092005 (2014)

16. K.W. Kim et al., Tests on $\mathrm{NaI}(\mathrm{Tl})$ crystals for WIMP search at the Yangyang Underground Laboratory. Astropart. Phys. 62, 249 (2014)

17. E. Shields et al., SABRE: a new NaI(Tl) dark matter direct detection experiment. Phys. Procedia 61, 169-178 (2015)

18. S. Cebrián et al., Background model for a $\mathrm{NaI}(\mathrm{Tl})$ detector devoted to dark matter searches. Astropart. Phys. 37, 60 (2012)

19. C. Cuesta et al., Analysis of the ${ }^{40} \mathrm{~K}$ contamination in $\mathrm{NaI}(\mathrm{Tl})$ crystals from different providers. Int. J. Mod. Phys. A 29, 1443010 (2014)

20. C. Cuesta et al., Slow scintillation time constants in $\mathrm{NaI}(\mathrm{Tl})$ for different interacting particles. Opt. Mat. 36, 316 (2013)

21. C. Cuesta et al., Bulk NaI(Tl) scintillation low energy events selection with the ANAIS-0 module. Eur. Phys. J. C 74, 3150 (2014)

22. J. Amaré et al., Study of scintillation in natural and synthetic quartz and methacrylate. Opt. Mat. 36, 1408 (2014)

23. J. Amaré et al., ANAIS: status and prospects. In Proceedings of RICAP2014. arXiv:1501.00104v1 [physics.ins-det] (2015)

24. C. Cuesta, ANAIS-0: feasibility study for a $250 \mathrm{~kg} \mathrm{NaI}(\mathrm{Tl})$ dark matter search experiment at the Canfranc Underground Laboratory. $\mathrm{PhD}$ thesis, Universidad de Zaragoza (2013)

25. E. Aprile et al., Study of the electromagnetic background in the XENON100 experiment. Phys. Rev. D 83, 082001 (2011) 
26. H.M. Araujo et al., Radioactivity backgrounds in ZEPLIN-III. Astropart. Phys. 35, 495 (2012)

27. E. Armengaud et al., Background studies for the EDELWEISS dark matter experiment. Astropart. Phys. 47, 1 (2013)

28. M. Agostini et al., The background in the neutrinoless double beta decay experiment GERDA. Eur. Phys. J. C 74, 2764 (2014)

29. D.S. Akerib et al., Radiogenic and muon-induced backgrounds in the LUX dark matter detector. Astropart. Phys. 62, 33 (2015)

30. J.B. Albert et al., Investigation of radioactivity-induced backgrounds in EXO-200. Phys. Rev. C 92, 015503 (2015)

31. V.A. Kudryavtsev, M. Robinson, N.J.C. Spooner, The expected background spectrum in NaI dark matter detectors and the DAMA result. Astropart. Phys. 33, 91 (2010)

32. R. Bernabei et al., Technical aspects and dark matter searches. J. Phys. Conf. Ser. 203, 012040 (2010)

33. J. Amaré et al., Cosmogenic radionuclide production in $\mathrm{NaI}(\mathrm{Tl})$ crystals. JCAP 02, 046 (2015)

34. J. Amaré et al., Preliminary results of ANAIS-25. Nucl. Instrum. Meth. A 742, 197 (2014)

35. J. Amaré et al., Background analysis and status of the ANAIS dark matter project. AIP Conf. Proc. 1672, 100001 (2015)

36. J. Amaré et al., Status of the ANAIS dark matter project at the Canfranc Underground Laboratory. In Proceedings of the Patras workshop 2015 (2015). arXiv:1508.07213v1 [physics.ins-det]

37. M.A. Oliván, Design, scale-up and characterization of the data acquisition system for the ANAIS dark matter experiment. PhD thesis, Universidad de Zaragoza (2016). arXiv:1601.07312 [physics.ins-det]

38. R. Bernabei et al., The DAMA/LIBRA apparatus. Nucl. Instrum. Meth. A 592, 297 (2008)

39. P. Adhikari et al., Understanding internal backgrounds of $\mathrm{NaI}(\mathrm{Tl})$ crystals toward a $200 \mathrm{~kg}$ array for the KIMS-NaI experiment. Eur. Phys. J. C 76, 185 (2016)
40. Decay Data Evaluation Projet. http://www.nucleide.org/DDEP WG/DDEPdata.htm

41. S. Agostinelli et al., Geant4 a simulation toolkit. Nucl. Instrum. Meth. A 506, 250 (2003)

42. R. Bernabei et al., Performances of the new high quantum efficiency PMTs in DAMA/LIBRA. JINST 7, P03009 (2012)

43. D.M. Mei et al., Cosmogenic production as a background in searching for rare physics processes. Astropart. Phys. 31, 417 (2009)

44. C. Zhang et al., Cosmogenic activation of materials used in rare event search experiments (2016). arXiv:1603.00098v1 [physics.ins-det]

45. J. Amaré et al., Production and relevance of cosmogenic radionuclides in NaI(Tl) crystals. AIP Conf. Proc. 1672, 140001 (2015)

46. W.C. Pettus, Cosmogenic activation in Nal detectors for dark matter searches. PhD thesis, University of Wisconsin-Madison (2015)

47. National Institute of Standards and Technology. http://physics.nist. gov/PhysRefData/Star/Text/ASTAR.html

48. N.J.T. Smith et al., A possible mechanism for anomalous pulses observed in sodium iodide crystals. Phys. Lett. B 485, 9-15 (2000)

49. S. Cooper et al., Radon-implanted ${ }^{214} \mathrm{~Pb}$ and anomalous pulses in sodium iodide detectors for dark matter. Phys. Lett. B 490, 6-8 (2000)

50. M. Clemenza et al., Radon-induced surface contaminations in low background experiments. Eur. Phys. J. C 71, 1805 (2011)

51. V. Alvarez et al., Radiopurity control in the NEXT-100 double beta decay experiment: procedures and initial measurements. JINST 8, T01002 (2013)

52. I. Lawson, B. Clevenland, Low background counting at SNOLAB. AIP Conf. Proc. 1338, 68 (2011)

53. X. Li et al., Simulation of natural radioactivity backgrounds in the JUNO central detector. Chin. Phys. C 40, 026001 (2016) 